\title{
A High Frequency Geometric Focusing Transducer Based on 1-3 Piezocomposite for Intravascular Ultrasound Imaging
}

\author{
Xiaohua Jian, Zhile Han, Pengbo Liu, Jie Xu, Zhangjian Li, Peiyang Li, \\ Weiwei Shao, and Yaoyao Cui \\ Suzhou Institute of Biomedical Engineering and Technology, Chinese Academy of Sciences, Suzhou, China \\ Correspondence should be addressed to Xiaohua Jian; simplejane1982@gmail.com and Yaoyao Cui; cuiyy@sibet.ac.cn
}

Received 2 June 2017; Accepted 2 August 2017; Published 5 September 2017

Academic Editor: Yongjin Zhou

Copyright (c) 2017 Xiaohua Jian et al. This is an open access article distributed under the Creative Commons Attribution License, which permits unrestricted use, distribution, and reproduction in any medium, provided the original work is properly cited.

\begin{abstract}
Due to the small aperture of blood vessel, a considerable disadvantage to current intravascular ultrasound (IVUS) imaging transducers is that their lateral imaging resolution is much lower than their axial resolution. To solve this problem, a single-element, $50 \mathrm{MHz}, 0.6 \mathrm{~mm}$ diameter IVUS transducer with a geometric focus at $3 \mathrm{~mm}$ was proposed in this paper. The focusing transducer was based on a geometric-shaped 1-3 piezocomposite. The impedance/phase, pulse echo, acoustic intensity field, and imaging resolution of the focusing transducer were tested. For comparison, a flat IVUS transducer with the same diameter and 1-3 piezocomposite was made and tested too. Compared with their results, the fabricated focusing transducer exhibits broad bandwidth (107.21\%), high sensitivity $(404 \mathrm{mV})$, high axial imaging resolution $(80 \mu \mathrm{m})$, and lateral imaging resolution $(100 \mu \mathrm{m})$. The experimental results demonstrated that the high frequency geometric focusing piezocomposite transducer is capable of visualizing high axial and lateral resolution structure and improving the imaging quality of related interventional ultrasound imaging.
\end{abstract}

\section{Introduction}

Intravascular ultrasound (IVUS) allows us to see a coronary artery from the inside-out, which has evolved to an important research tool of modern invasive cardiology [1]. In order to get high resolution image, an IVUS transducer usually has a high center frequency (20 60 MHz) [2], like $20 \mathrm{MHz}$ IVUS (Eagle Eye, Volcano Corporation), $40 \mathrm{MHz}$ IVUS (OptiCross, Boston Scientific), and $60 \mathrm{MHz}$ IVUS (Kodama, ACIST Medical Systems). A limitation to current IVUS transducer is that their lateral imaging resolution $(200 \sim 300 \mu \mathrm{m})$ is much lower than their axial resolution $(40 \sim 100 \mu \mathrm{m})[3,4]$. This is mainly caused by its small dimension $[5,6]$, which is extremely limited by the blood vessel.

Usually higher frequency transducer can provide higher lateral resolution, but it will cause a higher attenuation and decrease penetration capability. For example, to get $100 \mu \mathrm{m}$ lateral resolution at $3 \mathrm{~mm}$, the central frequency of a $0.6 \mathrm{~mm}$ diameter IVUS transducer needs to be more than $100 \mathrm{MHz}$, so it is not a very efficient method. Another possible method to a single-element IVUS transducer is synthetic aperture focusing, which has been shown to be able to improve the imaging resolution and SNR outside focus area by focusing the received signal from several emissions for rotating movements [7]. But it requires large sum data processing and decreases the imaging frame rate. Therefore, it is beneficial to try focusing transducer to get high lateral resolution IVUS image. The traditional method is to use acoustic lens, but fabricating a suitable acoustic lens to focus the ultrasound on IVUS is particularly challenging, since the IVUS catheter outer diameter is limited in the range of $3 \mathrm{~F} \sim 9 \mathrm{~F}(1 \mathrm{~mm} \sim$ $3 \mathrm{~mm}$ ), and the focusing length should be smaller than the coronary artery diameter $(3 \sim 5 \mathrm{~mm})$.

Therefore, self-focusing transducer will be a good choice. Fresnel Half-Wave-Band sources method was widely used for self-focusing, but its radius will be larger than $3 \mathrm{~mm}$ for getting better performance with enough loops at $20 \sim 60 \mathrm{MHz}$ [8]. One possible method is to fabricate high frequency PMN-PT single crystal focusing transducer by a mechanical dimpling technique. The reported dimpled $30 \mathrm{MHz}$ single crystal focusing transducer with a diameter of $1.6 \mathrm{~mm}$ can prove $139 \mu \mathrm{m}$ lateral resolution [9]. Similarly, oblong-shaped focused IVUS transducers using PZT were also able to 
TABLE 1: The design parameters of 1-3 piezocomposite.

\begin{tabular}{lcccc}
\hline Frequency & $\begin{array}{r}\text { Kerf } \\
\text { width }\end{array}$ & $\begin{array}{r}\text { Pillar } \\
\text { width }\end{array}$ & $\begin{array}{c}\text { Composite } \\
\text { thickness }\end{array}$ & $\begin{array}{c}\text { Volume } \\
\text { fraction }\end{array}$ \\
\hline $50 \mathrm{MHz}$ & $12 \mu \mathrm{m}$ & $18 \mu \mathrm{m}$ & $33 \mu \mathrm{m}$ & $36 \%$ \\
\hline
\end{tabular}

improve the lateral resolution [10]. Angled-focused singleelement transducer was another choice to improve IVUS lateral imaging resolution. As reported, the lateral resolution was improved from $270 \mu \mathrm{m}$ to $120 \mu \mathrm{m}$ with the angledfocused $45 \mathrm{MHz}$ PMN-PT single-element transducer [11]. But the single crystal or PZT are fragile and easy to crack in the process, which would affect the transducer performance and yield, while 1-3 piezocomposite will be an alternative choice for the active material, which can have high frequency, low acoustic impedance, and wide bandwidth [12, 13]. It has been reported for IVUS imaging and other high frequency endoscopic ultrasound imaging research [14-16]. There are many advantages to use a 1-3 piezocomposite as the IVUS transducer active substrate. First of all, the acoustic impedance of a 1-3 piezocomposite is significantly lower than common pure bulk ceramic, which can effectively decrease the acoustic mismatch between transducer and tissue and avoid the need for more matching layers $[17,18]$. Secondly, the electromechanical coupling coefficient of 1-3 composite is much higher than common piezoelectric ceramic, which is helpful to improve the imaging sensitivity. Furthermore, 1-3 piezocomposite consists of a large percentage epoxy, which made it easy to be geometrically shaped. Therefore, 1-3 piezocomposite was suitable for making geometric focusing IVUS transducer. Even in an early patent, the related proposal has been described [14]. Therefore, in this paper we proposed a geometric focusing $50 \mathrm{MHz}$ piezocomposite transducer for intravascular ultrasound imaging. For comparing, a flat IVUS transducer with the same 1-3 piezocomposite was also fabricated and tested following the same experiments; the results are described below in more detail.

\section{Design and Fabrication}

The focal length of the transducer was designed to $3 \mathrm{~mm}$, which is close to the natural focus $F$ corresponding to the near field range of flat IVUS transducer [5]:

$$
F=\frac{D^{2}}{4 \lambda},
$$

where the diameter $D$ of the transducer is $0.6 \mathrm{~mm}$, the wavelength $\lambda$ is about $30 \mu \mathrm{m}$ in our situation, and then the natural focal length of flat IVUS transducer is about $3 \mathrm{~mm}$.

The first step in the fabrication procedure was to make a high frequency 1-3 piezocomposite. Considering our laboratory experimental facilities, the design parameters of the 1-3 composite were listed in Table 1.

A two-dice and filling process was applied to fabricate this 1-3 composite. Firstly, a grid pattern was diced into a ceramic PZT-5H with $500 \mu \mathrm{m}$ thickness using a $12 \mu \mathrm{m}$ dicing saw. The depth of cuts is about $120 \mu \mathrm{m}$, and the pitch is $60 \mu \mathrm{m}$.

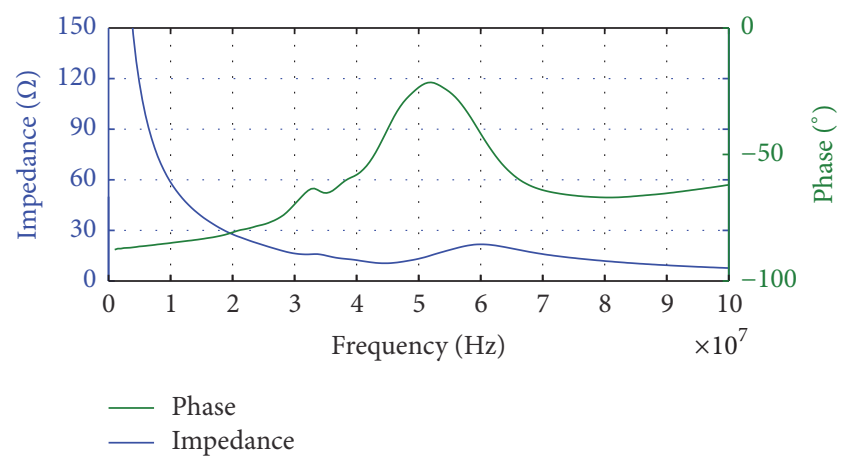

FIGURE 1: The measured impedance/phase of fabricated 1-3 piezocomposite.

The cuts were then filled with epoxy (Epo-Tek 301-2, Epoxy Technologies, Billerica, MA). After curing, the second set of cuts was made through the center of the PZT-5H pillars to create a composite pattern with $18 \mu \mathrm{m}$ pillars and $12 \mu \mathrm{m}$ kerfs as designed. The composite was filled and cured with EpoTek 301-2 again and then lapped to $33 \mu \mathrm{m}$ thick. After that, a $200 \AA$ A chrome/gold layer as the electrode was sputtered.

The electrical impedance of fabricated 1-3 piezocomposite was measured with an impedance analyzer E4991A (1 MHz $3 \mathrm{GHz}$, Agilent Technologies, USA), just as Figure 1 shows. The measured center frequency is $52.76 \mathrm{MHz}$.

Its electromechanical coupling coefficient $K_{t}$ can be calculated as [15]

$$
K_{t}=\sqrt{\frac{\pi}{2} \times \frac{f_{s}}{f_{p}} \times \tan \left(\frac{\pi}{2} \times \frac{f_{p}-f_{s}}{f_{p}}\right)},
$$

where $f_{p}$ is the parallel resonant frequency at which the resistance reaches the maximum and $f_{s}$ is the resonant frequency at which the conductance reaches the minimum. For our sample, $f_{s}$ is $44.05 \mathrm{MHz}$ and $f_{p}$ is $60.36 \mathrm{MHz}$, so according to (2), $K_{t}$ is about 0.70 , which was higher than pure bulk ceramic ( 0.5).

In the shaping process, the composite was firstly heated at $60^{\circ} \mathrm{C}$ for 1 hour to make it more flexible and then quickly mounted on a $3 \mathrm{~mm}$ radius PTFE ball with wax. The PTFE ball was used as the curving jig, which decides the focal length of the transducer. Conductive silver epoxy E-Solder 3022 was applied to the composite as the backing material in a PDMS (polydimethylsiloxane) mold. After curing at room temperature, the sample was heated to remove the PTFE ball. Then the sample was diced into the size of $0.6 \mathrm{~mm} \times 0.6 \mathrm{~mm}$. The individual piece was placed in a $1.2 \mathrm{~mm}$ diameter needle housing; the center core and mesh wire of a coaxial wire were connected to the piezocomposite surface and backing layer, respectively, with silver conductive. The gap between the transducer and the stainless steel needle was filled in by an insulating epoxy. At last, a $9 \mu \mathrm{m}$ Parylene $\mathrm{C}$ layer was coated as its matching layer. The final fabricated focusing transducer was shown in Figure 2. 


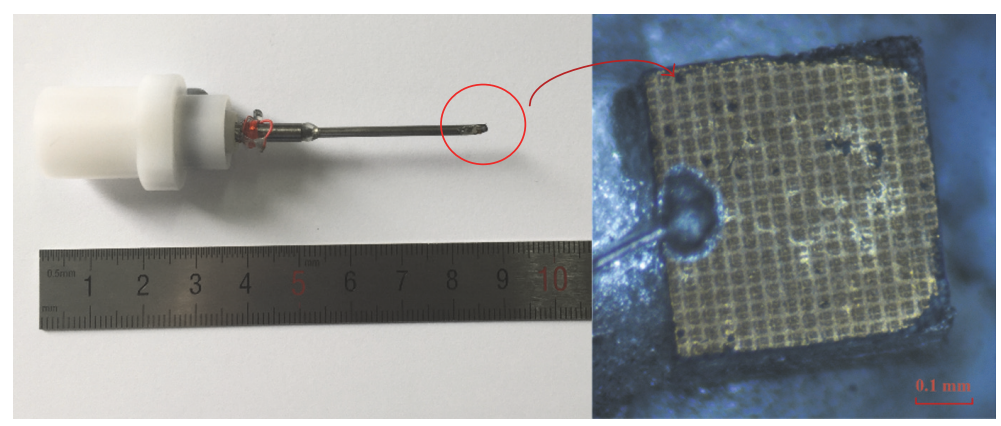

FIGURE 2: The fabricated focusing IVUS transducer.

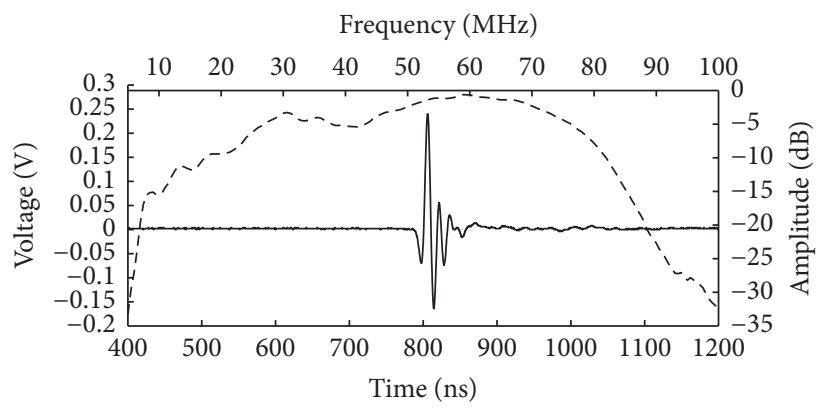

(a)

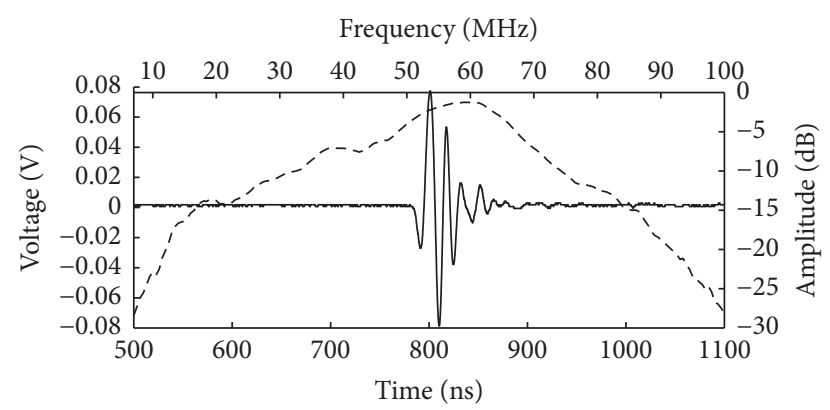

(b)

FIGURE 3: Pulse echo response and spectrum of (a) fabricated focusing IVUS transducer and (b) fabricated flat IVUS transducer.

\section{Results and Discussion}

A DPR500 (pulse amplitude: $90 \mathrm{~V}$, gain: $0 \mathrm{~dB}$, filter: 5 $300 \mathrm{MHz}$, RPF: $200 \mathrm{~Hz}$, JSR Ultrasonics, USA) was used as the pulser-receiver to measure the center frequency, $-6 \mathrm{~dB}$ bandwidth, and pulse echo amplitude of the fabricated focusing transducer and flat transducer. The pulse echo response was measured by recording the reflection from a quart polyethylene plastics flat placed at $3 \mathrm{~mm}$ in front of the transducer. The measured center frequency is $51.78 \mathrm{MHz}$, the $-6 \mathrm{~dB}$ bandwidth is $107.21 \%$, and the pulse half width is 17.37 ns just as Figure 3(a) shows. And the transducer pulse echo amplitude was measured as $404 \mathrm{mV}$ with $50 \Omega$ coupling impedance setting. For comparison, a flat IVUS transducer (unfocused) with the same diameter $0.6 \mathrm{~mm}$ was fabricated. This flat transducer was composed of the same 1-3 piezocomposite material, a Parylene $\mathrm{C}$ matching layer, and E-Solder 3022 backing layer. According to its pulse echo waveform and corresponding frequency spectrum as shown in Figure 3(b), its measured center frequency, $-6 \mathrm{~dB}$ bandwidth, pulse half width, and amplitude were $53.77 \mathrm{MHz}$, 64.77\%, $29.86 \mathrm{~ns}$, and $156 \mathrm{mV}$, respectively.

The acoustic distribution was measured by a 3D scanning system UMS III (scan step resolution $0.001 \mathrm{~mm}$, Precision Acoustics Ltd., Dorchester, UK) with a specially calibrated HGL-0085 hydrophone (20 60 MHz), as shown in Figure 4.

The hydrophone measurement step size was $30 \mu \mathrm{m}$. The acoustic intensity distribution of this fabricated transducer along $z$-axis was measured as shown in Figure 5. For avoiding the collision between the tested hydrophone and transducer, the recorded data was beginning at $0.5 \mathrm{~mm}$ away from the transducer. The measured focal length along the axial direction is $2.98 \mathrm{~mm}$, which is very close to the designed focal length $3 \mathrm{~mm}$. While the intensity of a flat IVUS transducer oscillates sharply in the near field range, then because of the attenuation, it will decrease linearly with the distance.

Because the lateral resolution is determined by the beam width perpendicular to the direction of wave propagation in an imaging plane, the transducer's acoustic intensity distributions in the focal plane ( $X-Y$ plane) were scanned with UMS III. According to the focal plane result in Figure 6(a), the diameter of the focal point of the fabricated focusing IVUS transducer (at $-6 \mathrm{~dB}$ ) is about $100 \mu \mathrm{m}$, which is one-third of the flat IVUS transducer's $300 \mu \mathrm{m}$, just as Figure 6(b) shows. These results indicate that the focusing IVUS transducer can achieve a higher lateral imaging resolution than usual flat ones.

The imaging resolution was tested by our IVUS system [16]. The transducer was fixed on the top of a catheter, and the catheter is driven by a motor, which is in the IVUS catheter interface module (CIM), to do a rotary scan. The block diagram of CIM is shown in Figure 7(b). A singleelement rotary scanning catheter is utilized to fix and rotate the transducer, which can spin at a speed of 1800 RPM. In the CIM, a contactless coupler with a flatten transfer curve from 10 to $110 \mathrm{MHz}$ with an attenuation better than $-1 \mathrm{~dB}$ is designed and manufactured to transfer high frequency signals between rotary and stationary side. For each cycle the catheter have turned, a frame composed of 512 scan lines would be captured. 12 bits analog to digital converter rate with 


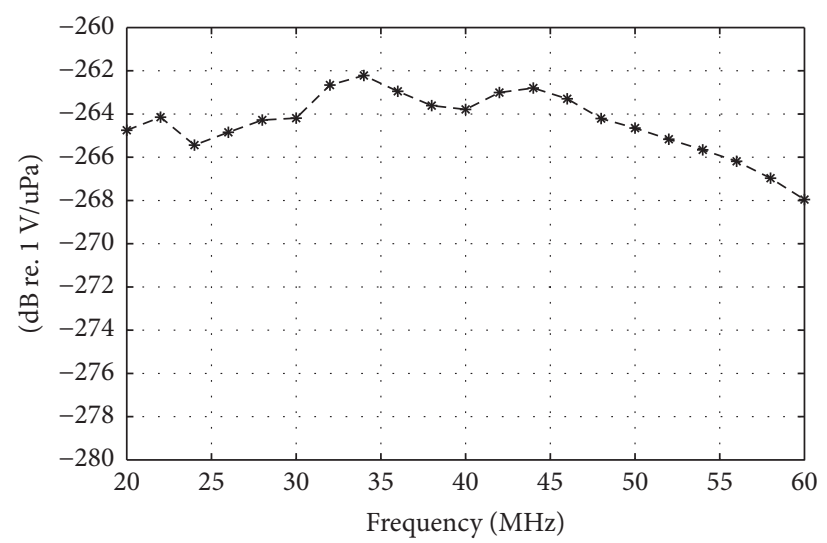

FIGURE 4: The response of HGL-0085 hydrophone with special calibration in the range of 20 60 MHz. Measurement uncertainty: 20 40 MHz, $2.2 \mathrm{~dB} ; 40 \sim 60 \mathrm{MHz}, 3.0 \mathrm{~dB}$.

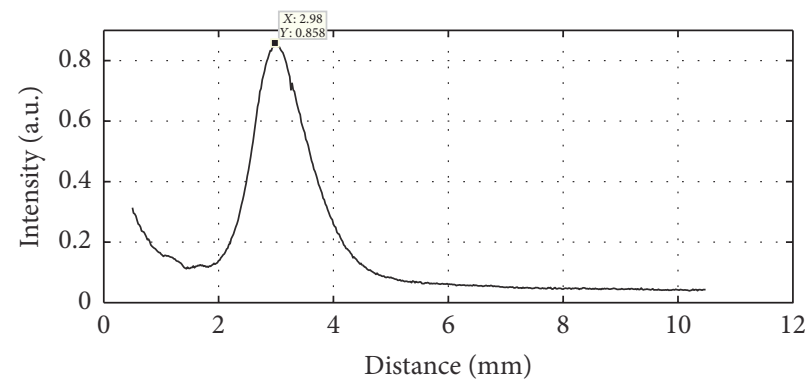

FIGURE 5: The acoustic intensity distribution of fabricated focusing IVUS transducer along $z$-axis.

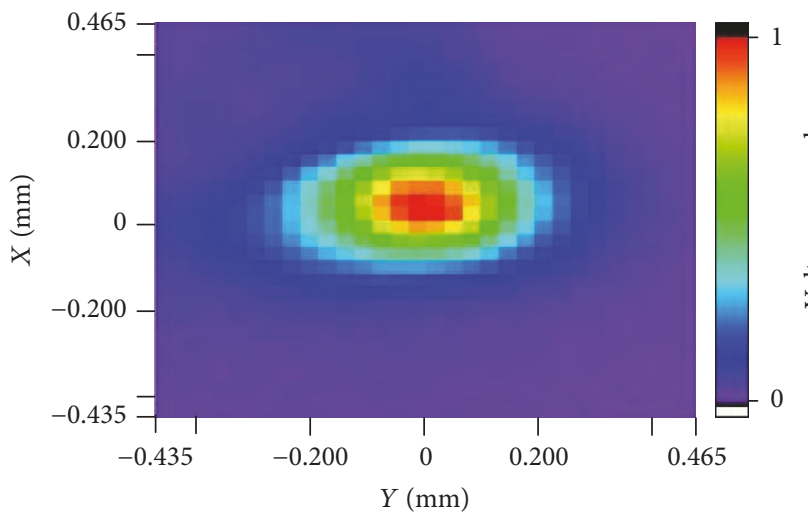

(a)

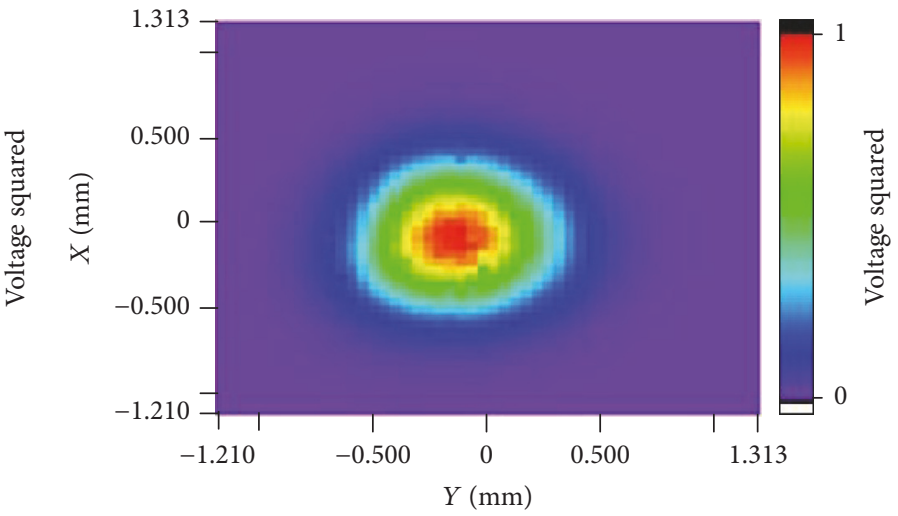

(b)

FIGURE 6: Measured acoustic intensity distribution of (a) fabricated focusing IVUS transducer; (b) fabricated flat IVUS transducer in the natural focus plane ( $X-Y$ plane).

a sampling rate of 220 MSPS ensure the echo information in enough frequency range could be recorded and provide a $25 \mathrm{fps}$ imaging speed. More details of our IVUS system can be found in our published [16].

The imaging targets were some resolution test jigs with fixed interval tungsten wires, including $50 \mu \mathrm{m}, 60 \mu \mathrm{m}$, $70 \mu \mathrm{m}, 80 \mu \mathrm{m}, 90 \mu \mathrm{m}, 100 \mu \mathrm{m}, 200 \mu \mathrm{m}, 300 \mu \mathrm{m}$, and $400 \mu \mathrm{m}$, just as Figure 8 shows. The diameter of tungsten wires is $10 \mu \mathrm{m}$. When testing the axial resolution, the transducer was adjusted to be perpendicular to the wire phantom, while for lateral resolution testing it will be parallel to the wire phantom, just as Figure 7(a) shows. For getting the best lateral resolution, the distance between the phantom wires and transducer was set to be $3 \mathrm{~mm}$ just as the focal length.

Figure 9 showed the lateral resolution testing results of the fabricated focusing and flat IVUS transducer, respectively. The bright arcs in the images represent the tungsten wires, while the center black hole and rings are caused by the transducer rotating. When using the $100 \mu \mathrm{m}$ interval tungsten wire phantom, the image of three tungsten wires is only a 


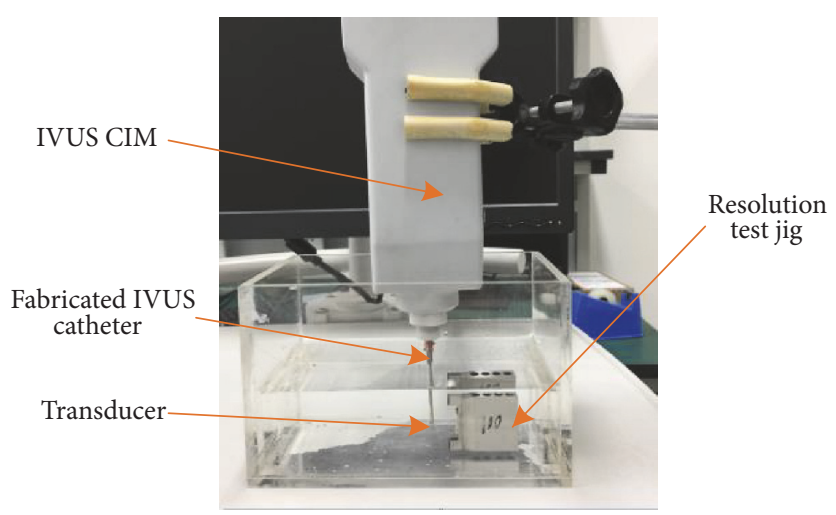

(a)

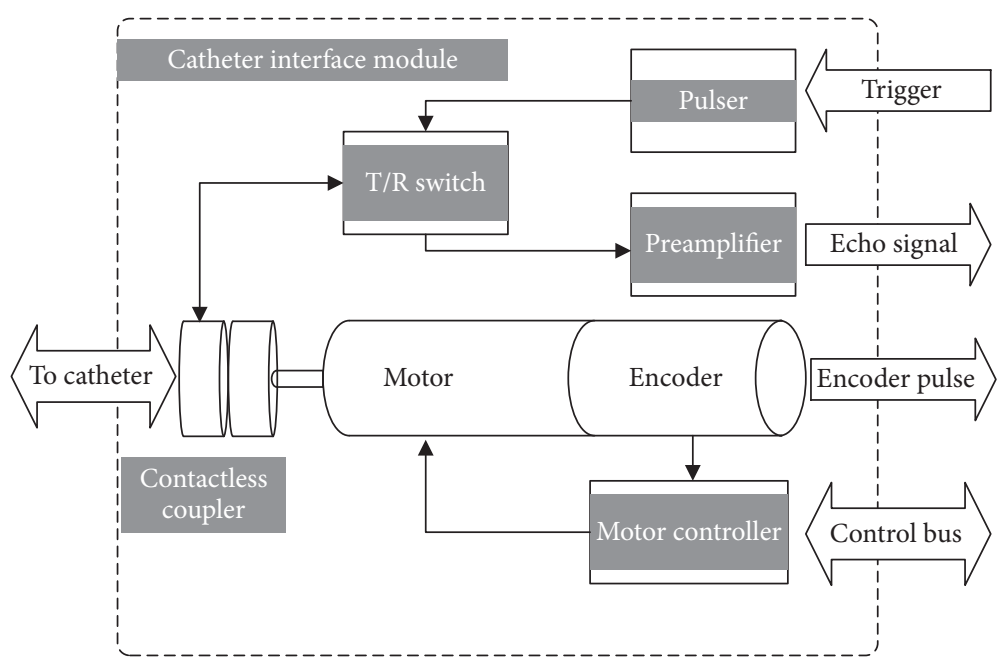

(b)

FIGURE 7: The imaging resolution test system: (a) experimental setup and (b) the block diagram of IVUS CIM (catheter interface module) [16].

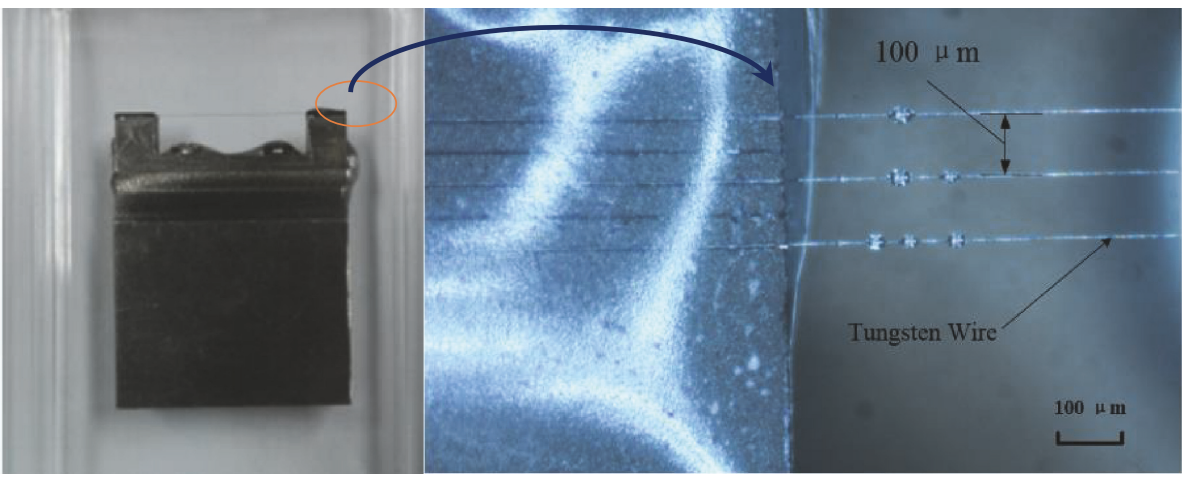

Figure 8: The imaging resolution test jig and tungsten wire phantom $(100 \mu \mathrm{m})$.

blurred curve in Figure 9(b) detected by the flat transducer. It is impossible to distinguish each line in this image. Until the $300 \mu \mathrm{m}$ interval tungsten wire phantom was used, the lines can be clearly separated in detected image in Figure 9(c). Therefore, the lateral resolution of flat IVUS transducer is tested as $300 \mu \mathrm{m}$.

In the same way, the lateral resolution of fabricated focusing IVUS transducer is tested as $100 \mu \mathrm{m}$, just as Figure 9(a) showed, which is three times of the flat IVUS transducer. That is because the lateral beam width is greatly reduced by adjusting the focal performance of focused transducer [19]. Therefore, it is beneficial to try focused transducer to get high lateral resolution IVUS image.

Similarly, Figures 10(a) and 10(b) showed the axial resolution testing images of the focusing and flat IVUS transducer, respectively. According to the results, the axial resolution of 


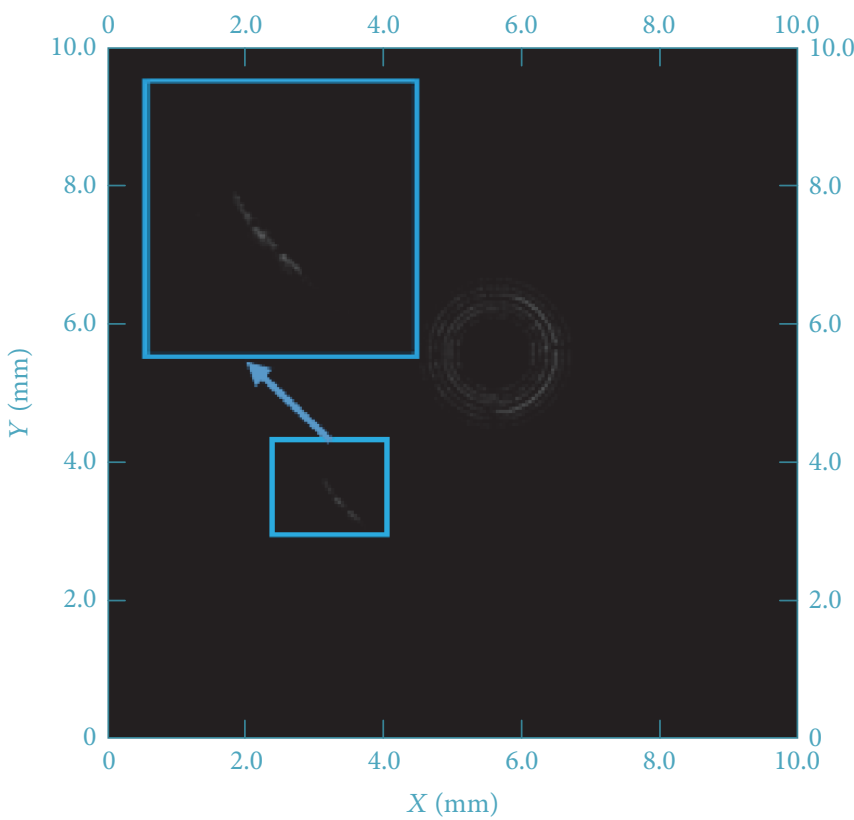

(a)

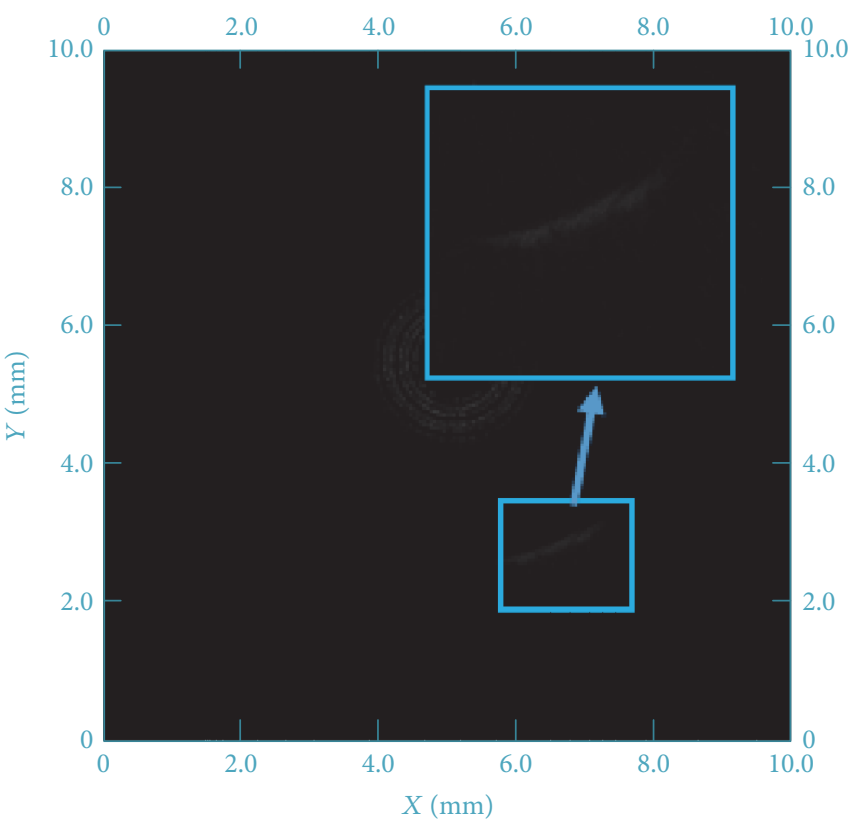

(b)

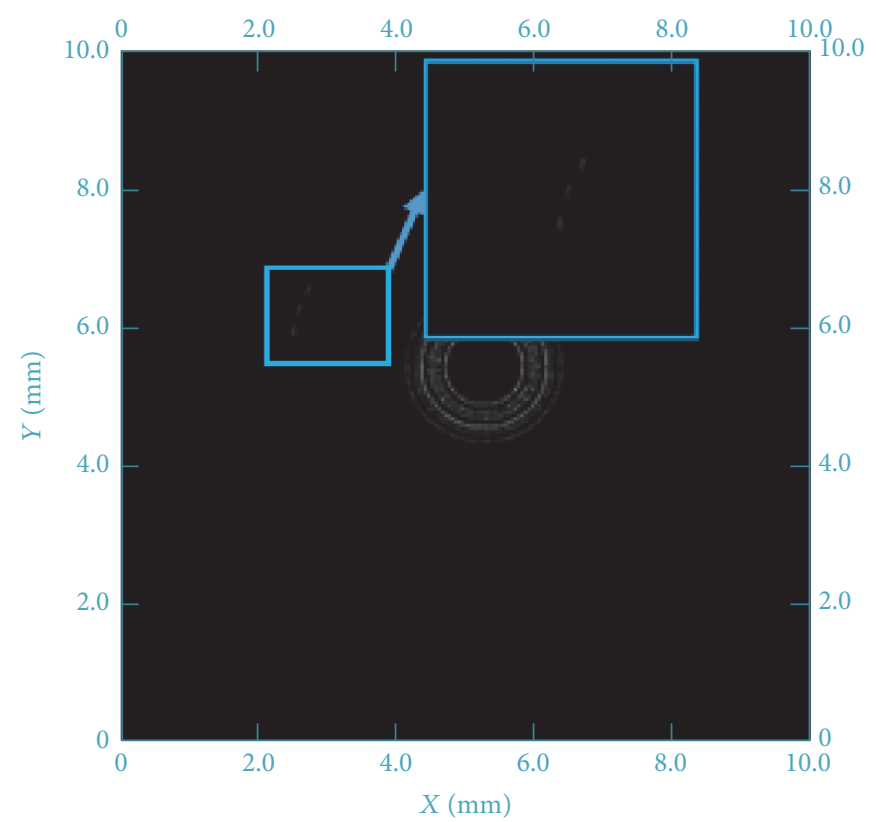

(c)

FIGURE 9: The lateral imaging resolution test images of (a) $100 \mu \mathrm{m}$ interval tungsten wire phantoms with focusing IVUS transducer; (b) $100 \mu \mathrm{m}$ interval tungsten wire phantoms with flat IVUS transducer; (c) $300 \mu \mathrm{m}$ interval tungsten wire phantoms with flat IVUS transducer.

focusing IVUS transducer is $80 \mu \mathrm{m}$, and the flat IVUS transducer is $80 \mu \mathrm{m}$ too. That is because the axial imaging resolution is mainly decided by center frequency of the transducer.

Furthermore, a hexagon hole phantom with $3 \mathrm{~mm}$ side length was used to test the imaging performance. The phantom was made by mixing $9 \mu \mathrm{m}$ silicon carbide ( $2 \%$ by weight) and $3 \mu \mathrm{m}$ aluminum oxide ( $2 \%$ by weight) with PDMS. When testing, the hexagon hole was filled with degas water, and the catheter distal with transducer was immersed into the hole. Figure 11 shows the phantom images detected by the fabricated focusing and flat IVUS transducer, respectively. Two images were both displayed in $12 \mathrm{~dB}$ dynamic range. Because of the high resolution and sensitivity, it is obvious that the image obtained by the focusing IVUS transducer was more clear and in a better shape. Particularly, the average contrast with respect to the locations of boundary and background in the images obtained by focusing and flat IVUS transducer were $-13.1 \mathrm{~dB}$ and $-16.2 \mathrm{~dB}$, respectively. 


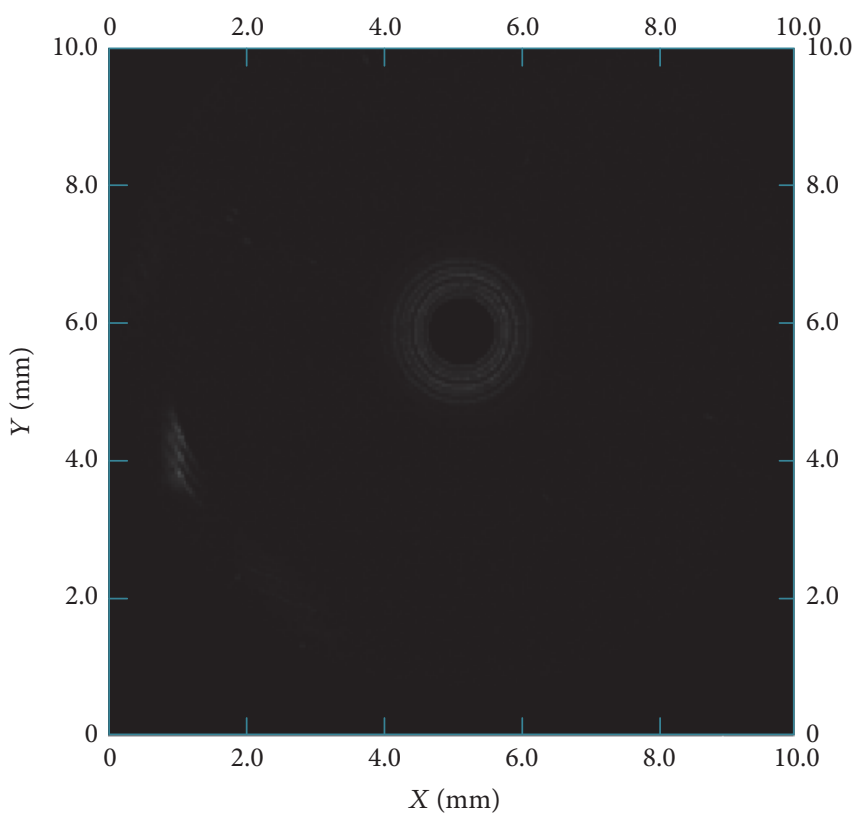

(a)

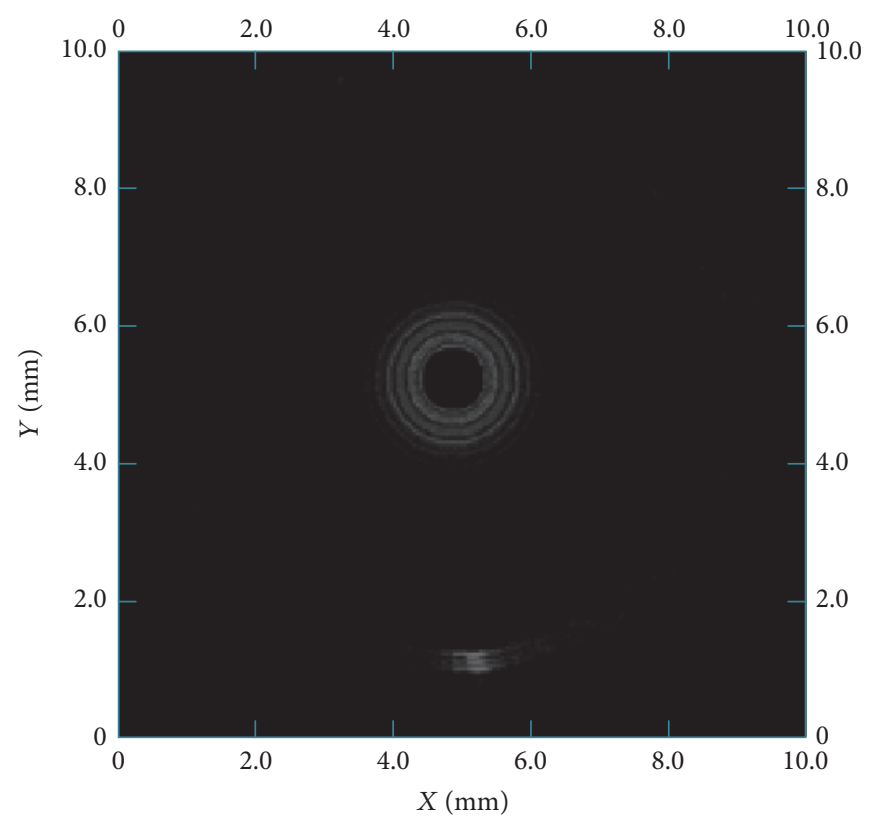

(b)

FIGURE 10: The axial resolution test images of $80 \mu \mathrm{m}$ interval tungsten wire phantoms with (a) focusing IVUS transducer and (b) flat IVUS transducer.

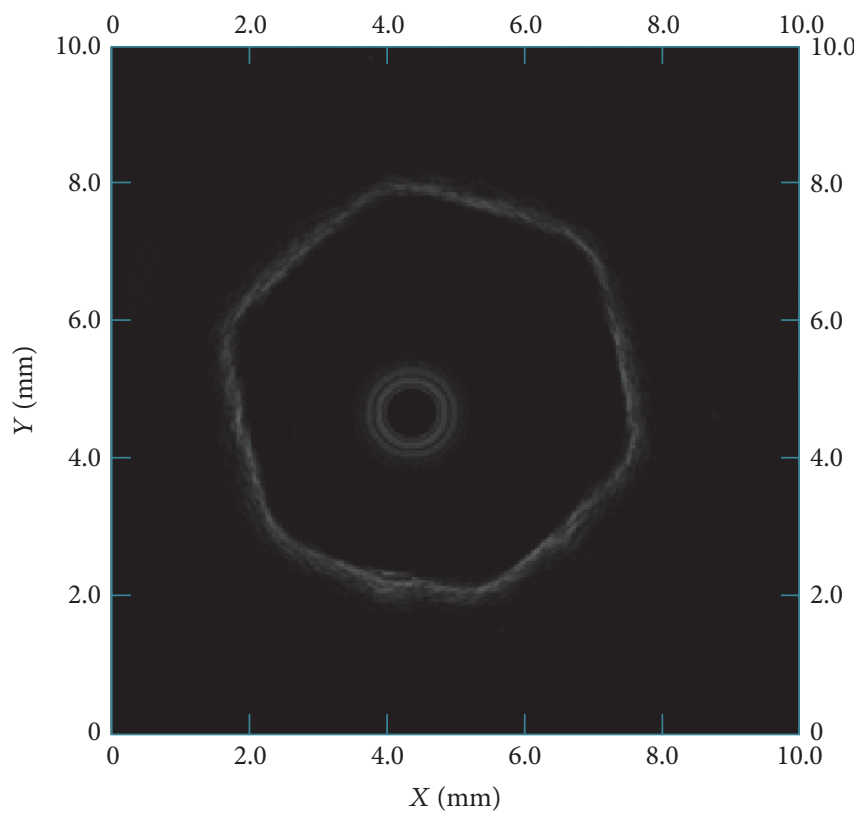

(a)

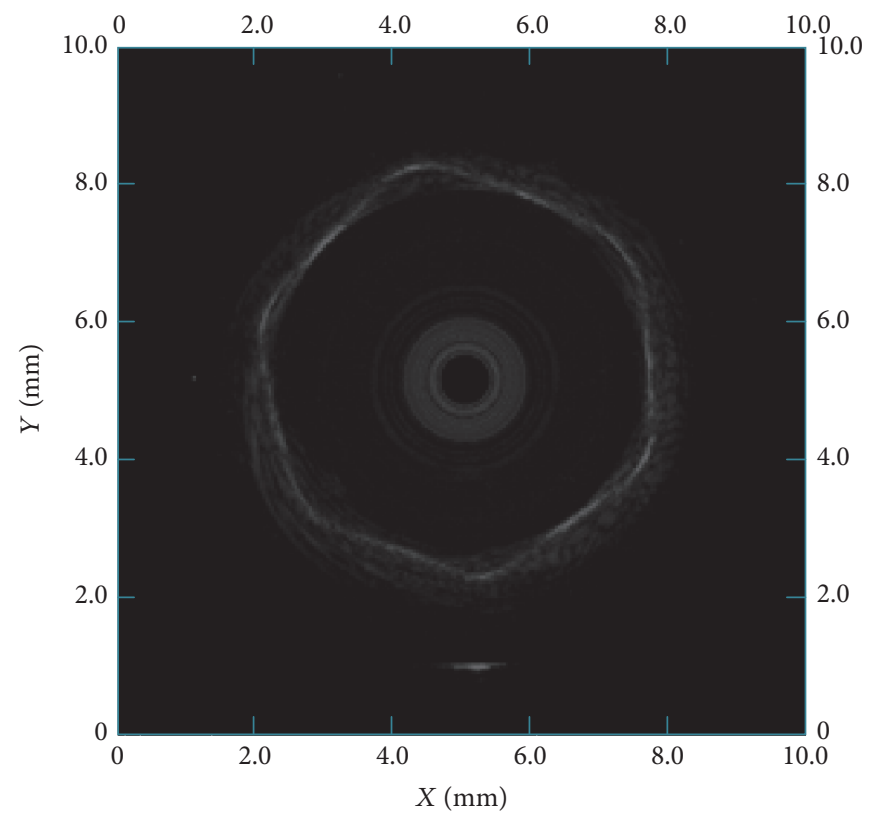

(b)

FIGURE 11: The image of hexagon phantom detected by (a) focusing IVUS transducer and (b) flat IVUS transducer.

At last, the main testing results were summarized in Table 2.

\section{Conclusion}

In this study, a micro high frequency IVUS transducer with spherical focusing was successfully produced using
PZT/epoxy 1-3 composite. The prototyped focusing IVUS transducer has a small size $(0.6 \times 0.6 \mathrm{~mm})$, short focal length $(3 \mathrm{~mm})$, and high lateral imaging resolution $(100 \mu \mathrm{m})$. The image obtained by our homemade IVUS system with the fabricated focusing transducer had a high signal to noise ratio and image quality. Based on these results, this micro high frequency focusing transducer has the potential for 
TABLE 2: The comparison of measured focusing and flat IVUS transducer performance.

\begin{tabular}{lcc}
\hline Properties & $\begin{array}{c}\text { Flat IVUS } \\
\text { transducer }\end{array}$ & $\begin{array}{c}\text { Focusing IVUS } \\
\text { transducer }\end{array}$ \\
\hline Center frequency & $53.77 \mathrm{MHz}$ & $51.78 \mathrm{MHz}$ \\
Aperture & $0.6 \mathrm{~mm}$ & $0.6 \mathrm{~mm}$ \\
Bandwidth & $64.77 \%$ & $107.21 \%$ \\
Echo peak & $156 \mathrm{mV}$ & $404 \mathrm{mV}$ \\
Axial resolution & $80 \mu \mathrm{m}$ & $80 \mu \mathrm{m}$ \\
Lateral resolution & $300 \mu \mathrm{m}$ & $100 \mu \mathrm{m}$ \\
\hline
\end{tabular}

intravascular ultrasound imaging and various high frequency endoscopic ultrasound imaging.

\section{Conflicts of Interest}

The authors declare that there are no conflicts of interest regarding the publication of this paper.

\section{Acknowledgments}

This work is supported by the National Key Technology Research and Development Program of the Ministry of Science and Technology of China (Grant nos. 2016YFC0103302 and 2017YFC0107202), International Cooperation Program of Jiangsu Province (Grant nos. BZ2016023 and BK20161235), the Funds for Technology of Suzhou, China (Grant nos. SYG201433, 201456, and SZS201510), and China Postdoctoral Program (Grant no. 2015M581409).

\section{References}

[1] B. N. Potkin, A. L. Bartorelli, J. M. Gessert et al., "Coronary artery imaging with intravascular high-frequency ultrasound," Circulation, vol. 81, no. 5, pp. 1575-1585, 1990.

[2] P. Schoenhagen, A. DeFranco, S. Nissen, and E. Tuzcu, IVUS Made Easy, CRC Press, 2005.

[3] F. Prati, E. Regar, G. S. Mintz et al., "Expert review document on methodology, terminology, and clinical applications of optical coherence tomography: physical principles, methodology of image acquisition, and clinical application for assessment of coronary arteries and atherosclerosis," European Heart Journal, vol. 31, no. 4, pp. 401-415, 2010.

[4] F. S. Foster, L. K. Ryan, and G. R. Lockwood, "High Frequency Ultrasound Scanning of the Arterial Wall," in Intravascular Ultrasound, Developments in Cardiovascular Medicine, pp. 91108, Springer Netherlands, 1993.

[5] J. S. Suri, D. L. Wilson, and S. Laxminarayan, Handbook of Biomedical Image Analysis, Springer US, Boston, Ma, USA, 2005.

[6] S. Rhee, "High frequency (IVUS) ultrasound transducer technology - Applications and challenges," in Proceedings of the 2007 16th IEEE International Symposium on the Applications of Ferroelectrics, ISAF, pp. 856-857, jpn, May 2007.

[7] H. Andresen, S. I. Nikolov, and J. A. Jensen, "Synthetic aperture focusing for a single-element transducer undergoing helical motion," IEEE Transactions on Ultrasonics, Ferroelectrics, and Frequency Control, vol. 58, no. 5, pp. 935-943, 2011.
[8] D. Huang and E. S. Kim, "Micromachined acoustic-wave liquid ejector," Journal of Microelectromechanical Systems, vol. 10, no. 3, pp. 442-449, 2001.

[9] Y. Chen, K. H. Lam, D. Zhou et al., "High frequency PMN-PT single crystal focusing transducer fabricated by a mechanical dimpling technique," Ultrasonics, vol. 53, no. 2, pp. 345-349, 2013.

[10] J. Lee, J. Jang, and J. H. Chang, "Oblong-Shaped-Focused Transducers for Intravascular Ultrasound Imaging," IEEE Transactions on Biomedical Engineering, vol. 64, no. 3, pp. 671-680, 2017.

[11] S. Yoon, J. Williams, B. J. Kang et al., "Angled-focused 45 $\mathrm{MHz}$ PMN-PT single element transducer for intravascular ultrasound imaging," Sensors and Actuators, A: Physical, vol. 228, pp. 16-22, 2015.

[12] X. Li, T. Ma, J. Tian, P. Han, Q. Zhou, and K. K. Shung, "Micromachined PIN-PMN-PT crystal composite transducer for high-frequency intravascular ultrasound (IVUS) imaging," IEEE Transactions on Ultrasonics, Ferroelectrics, and Frequency Control, vol. 61, no. 7, pp. 1171-1178, 2014.

[13] J. R. Yuan, X. Jiang, P.-J. Cao et al., "High frequency piezo composites microfabricated ultrasound transducers for intravascular imaging," in Proceedings of the 2006 IEEE International Ultrasonics Symposium, IUS, pp. 264-268, can, October 2006.

[14] J. Yuan, P. Cao, and R. Romley, "Piezocomposite transducers," 2006, Google Patents.

[15] A. Safari and E. K. Akdoǧan, "Piezoelectric and acoustic materials for transducer applications," Piezoelectric and Acoustic Materials for Transducer Applications, pp. 1-481, 2008.

[16] Y. Xiang, J. Xu, T. Lv, T. Gu, Z. Han, and Y. Cui, "A graphic processing unit based intravascular ultrasound (IVUS)," in Proceedings of the IEEE International Ultrasonics Symposium, IUS 2015, twn, October 2015.

[17] J. A. Brown, F. S. Foster, A. Needles, E. Cherin, and G. R. Lockwood, "Fabrication and performance of a $40-\mathrm{MHz}$ linear array based on a 1-3 composite with geometric elevation focusing," IEEE Transactions on Ultrasonics, Ferroelectrics, and Frequency Control, vol. 54, no. 9, pp. 1888-1894, 2007.

[18] X. Jiang, J. R. Yuan, A. Cheng et al., "Microfabrication of piezoelectric composite ultrasound transducers (PC-MUT)," pp. 918-921.

[19] Y. Saijo and A. F. W. Van der Steen, Vascular ultrasound, Springer Science and Business Media, 2003. 


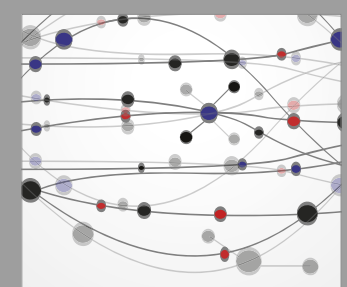

The Scientific World Journal
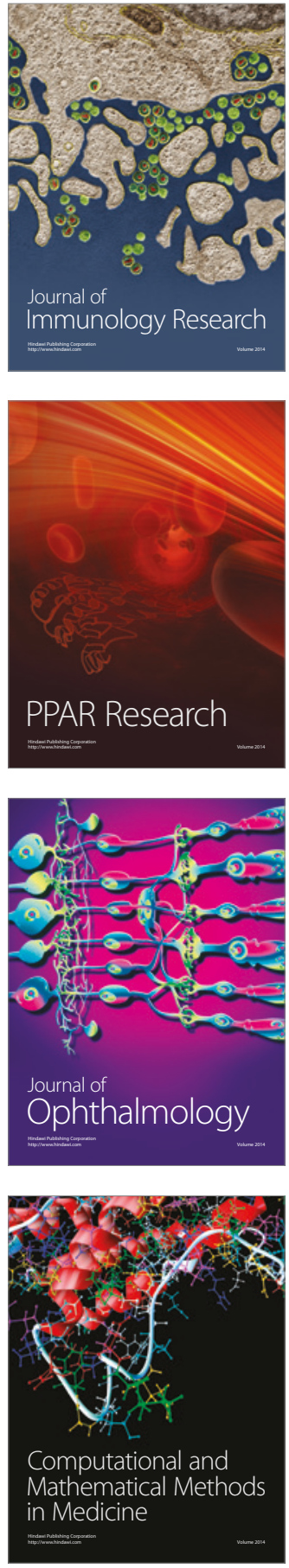

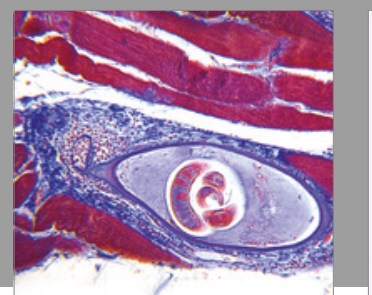

Gastroenterology Research and Practice
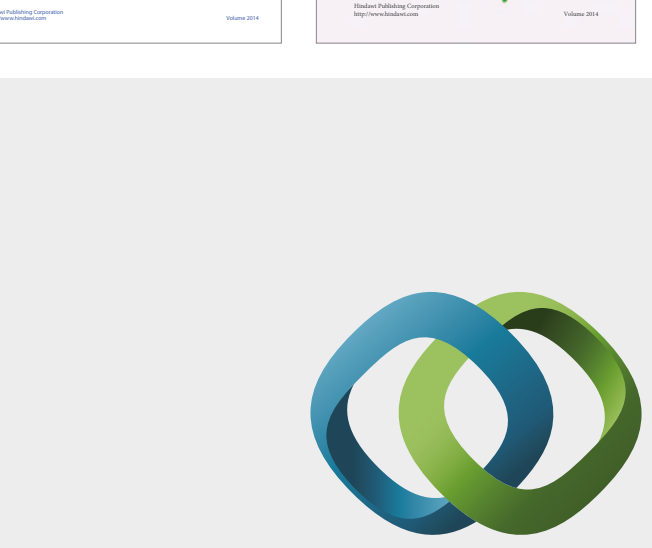

\section{Hindawi}

Submit your manuscripts at

https://www.hindawi.com
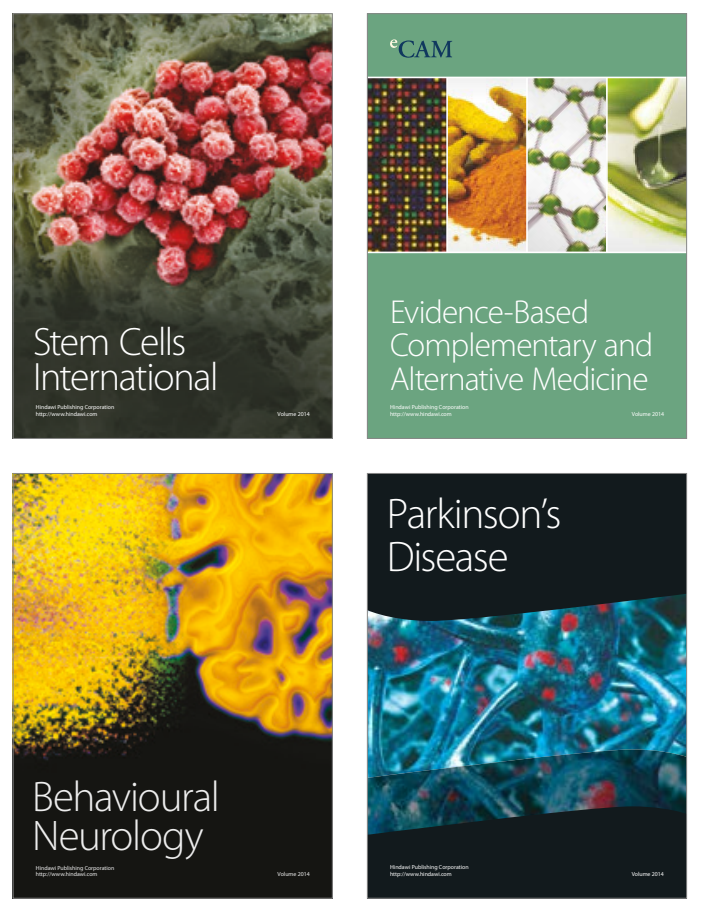
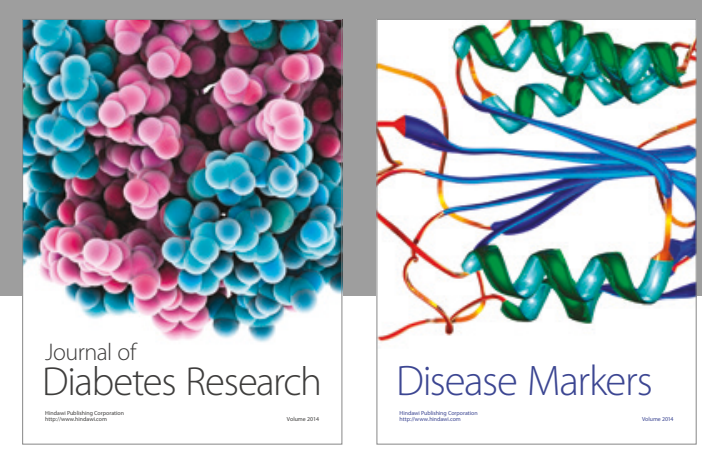

Disease Markers
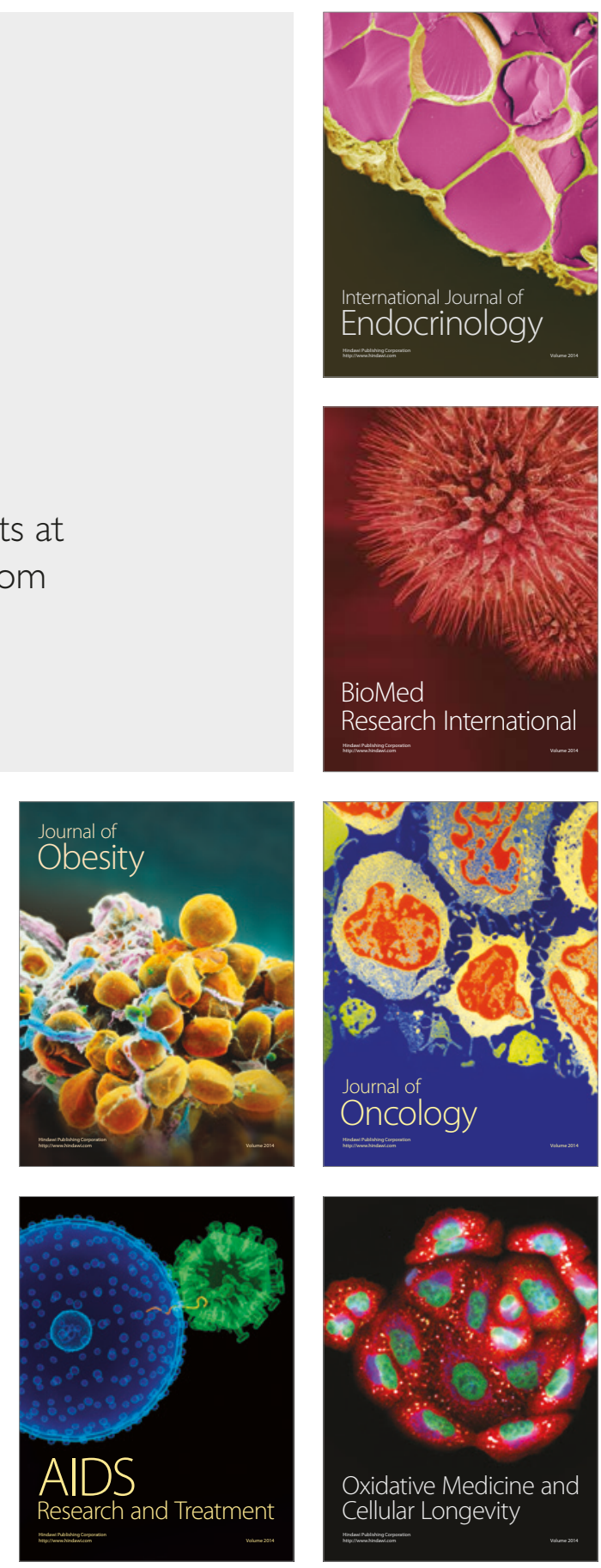